\title{
Structural insights into the insecticidal Vip3A toxin of Bacillus
}

4

5

\section{thuringiensis}

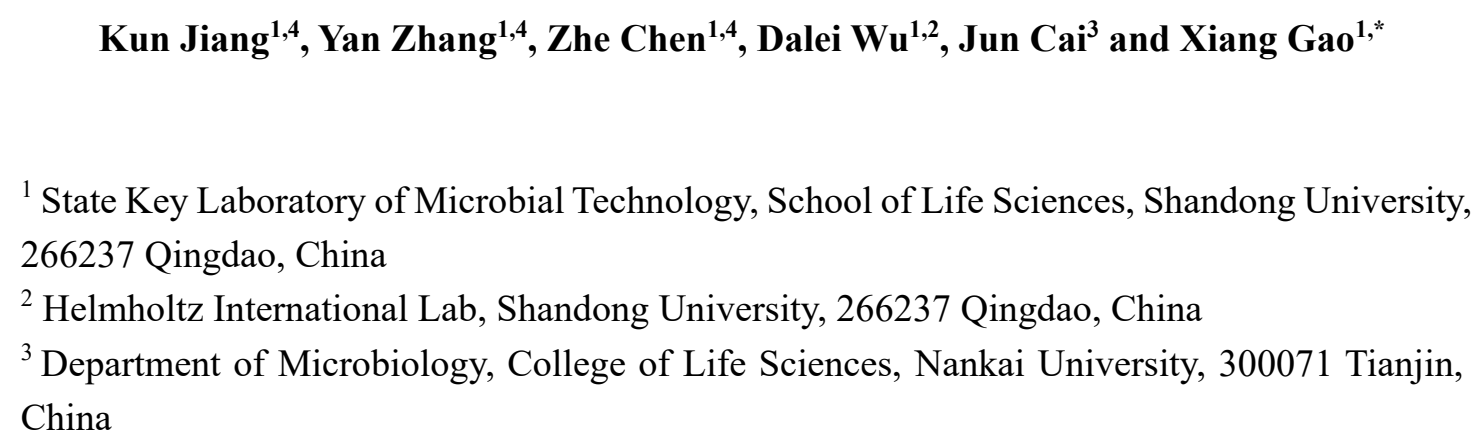


Abstract

The vegetative insecticidal proteins (Vips) secreted by Bacillus thuringiensis are regarded as the new generation of insecticidal toxins because they have different insecticidal properties compared with commonly applied insecticidal crystal proteins (Cry toxins). Vip3A toxin, representing the vast majority of Vips, has been used commercially in transgenic crops and bio-insecticides. However, the lack of both structural information of Vip3A and a clear understanding of its insecticidal mechanism at the molecular level, limits its further development and broader application. Here we present the first crystal structure of the Vip3A toxin in an activated form. Since all members of this insecticidal protein family are highly conserved, the structure of Vip3A provides unique insight into the general domain architecture and protein fold of the Vip3 family of insecticidal toxins. Our structural analysis reveals a four-domain organization, featuring a potential membrane insertion region, a receptor binding domain, and two glycan binding domains of activated Vip3A. We further identify the specific glycan moieties recognized by Vip3A through a glycan array screen. Taken together, these findings provide insights into the mode of action of Vip3 family of insecticidal toxins, and will boost the development of Vip3 into more efficient bioinsecticides. 


\section{Introduction}

The entomopathogenic bacteria Bacillus thuringiensis (Bt), is the most widely used microbial insecticide in the world ${ }^{1,2}$. It is renowned for its ability to produce insecticidal crystal proteins (Cry toxins) during its sporulation phase, which have been widely used in the prevention and control of agricultural pests through the development of transgenic plants or Bt-based biopesticides $^{3-5}$. However, many pests are not sensitive to Cry toxins, and the development of insect resistance to Cry toxins has also been reported ${ }^{1,6-8}$. The successful application of the Cry proteins, coupled with their limitations, has spurred on intensive research seeking to identify and characterize novel classes of insecticidal toxins that can be developed for agricultural purposes.

Vegetative insecticidal proteins (Vips), which are produced by Bt during its vegetative stages, have a wide spectrum of insecticidal activity, especially against lepidopteran pests ${ }^{9}$. To date, $\sim 150$ distinct Vip toxins have been identified, which have been classified into four families (Vip1, Vip2, Vip3 and Vip4) based on their sequence similarity ${ }^{10}$. Among the Vip toxin family, Vip3A toxins are the most abundant and most studied ${ }^{9}$. Compared with known Cry toxins, Vip3A toxins share no sequence homology, bind to different receptors ${ }^{11-14}$, and lack crossresistance ${ }^{15-18}$, therefore they are considered as ideal options to complement and expand the use of $\mathrm{Bt}$ in crop protection and resistance management. At present, the Vip3Aa toxin is the only family member that has been used in commercial transgenic crops together with Cry toxins, and no field-evolved resistance has yet been reported ${ }^{1,9,19}$. However, the lack of structural information and incomplete understanding of its mechanisms of action have severely limited the further development of Vip3A as a tool in pest control. 

terminus and a variable $\mathrm{C}$-terminal region. The $\sim 89 \mathrm{kDa}$ Vip3A protoxin could be digested by insect midgut juices into two fragments: a $\sim 20 \mathrm{kDa}$ fragment corresponding to the $\mathrm{N}$-terminal 198 amino acids, and a $\sim 66 \mathrm{kDa}$ fragment corresponding to the rest of Vip3A protein and carrying the toxic activity, which is considered to be the toxic core ${ }^{9,11,20-25}$. This processing step is regarded as an essential step for its activation and toxicity ${ }^{11,20,21,26-28}$. Since their discovery in $1996^{29}$, Vip3A proteins have been the subject of intensive research. It has been reported that Vip3A stimulates membrane pore formation and apoptosis upon binding to target cells, which is proposed to be responsible for its cytotoxic effects ${ }^{11,30,31}$. The scavenger receptor class C like protein (Sf-SR-C) and the fibroblast growth factor receptor (Fgfr) have been reported as potential receptors for Vip3 $\mathrm{A}^{13,14}$. However, although some in silico modeling efforts and low resolution cryo-EM structures have attempted to obtain structural insight for these toxins, the atomic structure of Vip3A is still not available, which makes it difficult to reveal the relationship between their structure and function ${ }^{27,28,32,33}$. a four-domain organization which is likely to be conserved for the toxic core of all insecticidal Vip3 family toxins. We identify conserved hydrophobic $\alpha$-helices in domain II, which we predict to be involved in the membrane insertion process that follows the activation of cellbound toxin. Structure-guided cell binding assays reveal that domain III is central to host cell targeting and binding of Vip3A toxins. Structural analysis and glycan array studies indicate that 
underlying mode of action of insecticidal Vip3 toxins.

\section{Results}

\section{Overall structure of Vip3Aa11 200 -end}

We used a Vip3A toxin from Bt strain C9, which has been named Vip3Aa11 (GenBank accession No. AY489126.1) in this study. Full-length Vip3Aa11 consists of 789 amino acids, which has been demonstrated to be digested between residues K198 and D199 by insect midgut juice $^{20,26,27}$. Of the two resulting fragments, the C-terminal fragment (D199 to end) is considered to be the toxic core of Vip3A. core. Using spare matrix crystallization screening, we only identified one condition that yielded needle-shaped crystals of Vip3Aa11 protoxin. However, the crystals diffracted to only $\sim 15 \AA$ and could not be improved despite extensive effort. No crystals were observed for the Vip3Aa toxic core construct despite screening more than 1000 crystallization conditions. However, when we deleted the N-terminal amino acid (Asp199) from the Vip3Aa11 toxic core, we

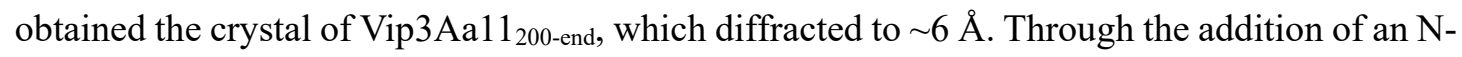
terminal MBP (Maltose Bind Protein) tag, we were able to isolate crystals with improved diffraction. values to 0.1980 and 0.2389 , respectively (Supplementary Table1). The crystal belongs to the 
determined that there was limited interaction between two dimers, indicating that their association was caused by the crystal packing (Supplementary Fig. 1). Notably, the two Vip3Aa11 molecules in the "dimer" showed moderate conformational variations, with a core root mean square deviation (r.m.s.d) of $1.234 \AA$ among $468 \mathrm{C} \alpha$ atoms (Supplementary Fig. 2). domains III, IV and V, but not for domain II (Supplementary Fig. 2), suggesting that domain II might potentially be involved in the conformational changes during the activation of Vip3A toxins. However, the result of gel filtration chromatography showed that MBP-Vip3Aa11 200-end mainly exists as a monomer in solution (Supplementary Fig. 3), which is consistent with observations from a previous study ${ }^{21}$. Therefore, we used the monomeric structure of MBPVip3Aa11 200-end for subsequent analysis. $1 \mathrm{a}$ and $\mathrm{b}$ ). Therefore, the protoxin can be divided into five domains, starting from $\mathrm{N}$-terminus: V, 679-789 in Vip3Aa11 (Fig. 1a and Supplementary Fig. 4). The overall structure of the Vip3Aa11 toxic core resembles a lobster, wherein domains II and III form the body, and domains IV and V are the claws of the lobster (Fig. $1 \mathrm{~b}$ and c). The connection between domain flexible loops, which indicates that the relative locations and orientations of these two domains

149 could change under different biological circumstances. There are over 100 known proteins of the Vip3 family. Based on their high degree of sequence conservation and previous studies ${ }^{9,20,}$ 
154 Domain II of Vip3Aa11 (residues199-327) consists of five helices, which form two layers (Fig.

2a). The outer layer facing the solution contains two short helices, $\alpha 2$ and $\alpha 3$, while the inner layer contacts with the upper portion of the inner layer and is almost perpendicular to the inner layer. An intensive search against the PDB (Protein Data Bank) through the DALI server ${ }^{35}$ failed to identify any known structure that shows significant homology with all five $\alpha$-helices of the domain II from Vip3Aa11, suggesting that this is a novel protein fold. 30 amino acid residues, starting from E267 at the N-terminus to L296 at the C-terminus. Electrostatic surface potentials analysis shows that the majority of charged and polar amino acid residues locate at $\mathrm{N}$-terminal and $\mathrm{C}$-terminal ends of helix $\alpha 4$ (Fig. 2b). For the middle portion of helix $\alpha 4$, from F274 to L289, 75\% amino acid residues are hydrophobic residues. Sequence alignment through Vip3 family shows that the hydrophobic region of helix $\alpha 4$ is very much conserved and it is also the most agminated hydrophobic region of Vip3 family proteins

171 to the hydrophobic helical hairpin from typical colicin fold, which has been shown to be

172 involved in membrane insertion of several $\alpha$-pore-forming toxins ${ }^{36}$. 

most conserved domain compared to other domains. Electrostatic surface potentials analysis shows that there is an obvious hydrophobic surface, which is mainly contributed by the conserved helix $\alpha 1$ and $\alpha 4$ (Fig. 2d). demonstrating the membrane insertion properties of Vip3A toxins ${ }^{13}$ collectively suggest that domain II is involved in the membrane insertion processes thought to be triggered once Vip3A binding to host cell receptors.

\section{Domain III is involved in receptor binding of Vip3A toxin}

Domain III of Vip3Aa11 (residues 328-518) consists of twelve $\beta$ strands and one short $\alpha$-helix similar "Greek-key" topology (Fig. 3a) with a hydrophobic center featuring highly conserved residues V349, F360, I362 and L370 from $\beta$ sheet I, I425 and F427 from $\beta$ sheet II and I481, to explore whether domain III serves as a receptor binding domain for Vip3A toxins. cells), which have previously shown to be specifically targeted by Vip3 toxins ${ }^{14,37}$. To determine 
binding assays using different C-terminal RFP-tagged Vip3Aa truncation derivatives (shown schematically in Fig. 3c). As shown in Fig 3d, 3e and Supplementary Fig. 6, while domains IV and $\mathrm{V}$ do not show detectable binding to Sf9 cells, the binding of a construct featuring only domains II and III to Sf9 cells is indistinguishable from that of full-length Vip3Aa. The interaction of domain III alone with Sf9 cells is significantly stronger than that of the domain I-II construct, indicating that domain III is central to Vip3A receptor binding to Sf9 cells.

\section{Domains IV and V are glycan binding motifs}

Domains IV and V both are all $\beta$ sheets folds (Fig. 4a and b). Unlike domains II and III, which have compact organization, domains III/IV and IV/V are connected by long and flexible loops

(Fig. 4a). In addition to these loops, there are several polar interactions between domains IV/V and domain III, that reduce the flexibility and fix the domains IV and V at the observed positions and orientations (Fig. 4a). the "jelly-roll" topology. Despite showing only 17\% sequence identity (Fig. 4c), domains IV

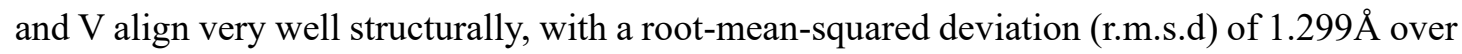
$61 \mathrm{C} \alpha$ atoms (Fig. 4d). To examine the potential function of these two domains, we searched for their structural homologues using the DALI $\operatorname{sever}^{35}$. The results for both domains show a very high similarity ( $\mathrm{Z}$ score $>10)$ to family 16 carbohydrate binding module (CBM16) of SLayer associated multidomain endoglucanase (RCSB ID 2ZEY). Superimposition of domains IV, $\mathrm{V}$ and CBM16 demonstrates that these three motifs share a similar fold (Fig 4e), suggesting 
215 the highly active mannanase from the thermophile Thermoanaerobacterium

216 polysaccharolyticum with high specificity toward $\beta 1,4$-glucose or $\beta 1,4$-mannose polymers ${ }^{38}$.

217 Analysis of the electrostatic surface potential shows that both domains IV and V have a surface

218 pocket at a similar position to a sugar-binding pocket of the CBM16 domain, although all three

219 pockets have different shapes and charge distributions (Fig. 5a). Taken together, our structural

220 analysis indicates that domains IV and V of Vip3A both contain a conserved glycan binding

221 motif and that these motifs may target different sugars.

In order to investigate the glycan binding properties of Vip3Aa11, we conducted glycan array screening with purified biotin-labelled, full-length Vip3Aa11. Screening results revealed that Vip3Aa11 has a strong binding preference to chitosan (GlcN $\beta 1-4 \mathrm{GlcN}$, structural component of the peritrophic matrix (PM), an extracellular envelope that surrounds the midgut of most insects ${ }^{39}$. Epithelial cells in the midgut of insects synthesize chitin as a homopolymer of $\beta-(1,4)-\mathrm{N}$-acetyl-D-glucosamine (GlcNAc), which may be subsequently converted to chitosan (a homopolymer of GlcN) by chitin deacetylase (CDA) secreted by insect midgut cells ${ }^{40}$. The identification of chitin and chitosan as targets of Vip3Aa is therefore consistent with previous studies that this toxin demonstrates specificity for binding insect bind to the sf9 cells which are from ovarian tissue and may lack of chitin and chitosan ${ }^{39,40}$. In addition to chitin and chitosan, the glycan array results revealed that Vip3Aa binds Lacto-N- 

relevance of Vip3A's glycan binding specificities.

\section{Discussion}

Vip3A toxins show a wide spectrum of specific insecticidal activities and are functionally distinct compared to the Cry toxins. These features makes them good candidates for combined application with Cry toxins in transgenic crops to broaden the insecticidal spectrum and to prevent or delay resistance ${ }^{1,9,14}$. The structural features and insecticidal mechanisms of Cry toxins have been studied in detail, which has been crucial to their widespread application ${ }^{2-6,19}$. However, despite of the fact that Vip3 toxins were identified almost 25 years ago ${ }^{29}$, their mode of action remains poorly understood. One of the main reasons for this is the lack of a highresolution three-dimensional structure, which significantly impedes detailed molecular level functional and mechanistic studies and thus limits the development of their insecticidal potential. Accordingly, many groups have made great efforts to obtain or predict the atomic structure of Vip3 $\mathrm{A}^{21,27,28,32,33}$. However, high-resolution crystal structure of Vip3 toxin family is still missing. In this study, we report the first crystal structure of the Vip3A toxin, which provides a badly-needed framework to explore the molecular-level functional details of Vip3family toxins.

Although the amino acid sequence similarity between the Vip3 family toxin and the three domain Cry family (3d-Cry) toxin is very low, our three-dimensional structural analysis showed interesting convergent evolution between these two families. Domain II of Vip3 has an all $\alpha$-helix fold, including two conserved hydrophobic $\alpha$-helices. Similarly, domain I of 3d-Cry 
also has an all $\alpha$-helix fold and two hydrophobic $\alpha$-helices, although it has additional $\alpha$ helices surrounding the conserved hydrophobic helices ${ }^{4,42}$. Several studies have reported that domain I of 3d-Cry toxin is involved in its membrane insertion and pore formation processes through its conserved hydrophobic $\alpha$-helices ${ }^{4,43-45}$. This therefore suggests that domain II of Vip3 may also take part in these processes through its conserved hydrophobic $\alpha$-helices. This is further supported by the fact that the insertion of the typical colicin fold, a hydrophobic helical hairpin, into the inner bacterial membrane is a minimal requirement for the pore formation of the poreforming colicins ${ }^{36}$. a conserved hydrophobic core. Extensive studies on domain II of 3d-Cry toxins showed that it plays a key role in the recognition of midgut receptors. The results of our cell binding assay indicate that Vip3 domain III is also central to cell binding. However, the receptor binding specificity of Vip3 toxins is still unclear and requires further studies. that all Vip3A proteins contain a carbohydrate-binding motif (CBM_4_9 superfamily;

274 pfam02018) in the C-terminus (amino acids 536 to 652 in Vip3Aa). The function of this glycan

275 binding domain is not yet clear ${ }^{9}$. In the present structure, we found that instead of the single 
279 to have different glycan binding specificities. This multiplicity of CBMs in Vip3 toxins may

280 increase the diversity of their target polysaccharides. Interestingly, our glycan array results

281 indicate that Vip3Aa is able to bind both chitin (and the related chitosan) as well as a spectrum

282 of different monosaccharides. It is tempting to speculate that each of these two classes of

283 glycans is specifically recognized by one of Vip3's two glycan-binding domains.

In conclusion, we find here that although the overall structure and domain organization

are very different between Vip3 toxin and 3d-Cry toxin, these two families are comprised of

functionally and structurally related modules that are assembled in different ways, which may

expand the insecticidal spectrum of Bt and make Bt more powerful and efficient to target and

kill their hosts.

During the preparation of this manuscript, Zheng et al. reported the crystal structure of

Vip3B2160 (the pro-toxin domain cleaved to produce the active core that we have crystalized 
I and II. We hypothesize that this structural difference in domain II between the full-length and cleaved Vip3 proteins may represent the conformational change that switches this toxin from "inactive" to "active". In this scenario, once domain I is cleaved by insect midgut juice, the $\alpha$ helices of domain II undergo a dramatic structural shift that enables helix $\alpha 1$ to rotate to form a hairpin-like structure with helix $\alpha 4$. This new conformation, which strongly resembles the colicin A-type membrane insertion fold, represents the active membrane insertion moiety of Vip3 toxins (Fig. 6c). In a word, our crystal structure of Vip3A toxic core, combined with the structure of full-length Vip3B, reveals that the cleavage between domain I and domain II of Vip3 toxin, and the further conformational changes of domain II are essential steps for Vip3's activation and function. the general domain organization of Vip3 family toxin and the potential function of each domain. Our study confirmed the glycan binding ability and defined the specificity of the Vip3A toxin using a glycan array screen. Importantly, we identify a conserved colicin A-type membrane insertion fold in the active core of our Vip3A structure which, through a structural comparison with full-length (inactive) Vip3B, enabled us to propose an activation mechanism for the Vip3 family toxins. Collectively, these data provide important structural and functional insights into Vip3 family toxins as well as a valuable resource to guide future studies and to re-evaluate the previous genetic and functional studies that will be crucial for the development of Vip3 as a new generation of bio-insecticides. 
Bacterial strains, cell lines and plasmids

E. coli BL21(DE3) for plasmid constructions and protein purification were cultured at $37^{\circ} \mathrm{C}$ in lysogeny broth (LB) or agar. Methionine auxotrophic E. coli strain B834 (DE3) (Novagen) were used for selenomethionine-substituted (SeMet) Vip3 $\mathrm{Aa}_{200-\text {-end }}$ expressing. The $S$. frugiperda Sf9 cells were maintained and propagated in Sf-900 II SFM (Invitrogen) culture medium at $27^{\circ} \mathrm{C}$. The DNA of Vip3Aa 200 -end was amplified from the Vip3Aa11 gene (GenBank accession No. AY489126.1) using oligonucleotide primer Vip200-F and Vip200-R and cloned into the pET28a vector with an N-terminal $6 \times$ His-MBP (maltose binding protein) tag. Plasmids used for RFP (red fluorescent protein) and C-terminal RFP tagged Vip3Aa (Vip3Aa-RFP) expression were constructed as described by Kun et $\mathrm{al}^{14}$. The different Vip3Aa truncations DNA were amplified from the Vip3Aa11 gene using oligonucleotide primer pairs; DmI-III-F and DmI-IIIrespectively. All plasmids were generated by the Gibson assembly strategy ${ }^{51}$. The nucleotide sequences of recombinant plasmid were verified by DNA sequencing. All the primers used in this study are shown in Supplementary Table 3.

\section{Protein expression and purification}

339 Native His-MBP-Vip3Aa 200 -end (Vip3 $\mathrm{Aa}_{200-\text {-nd }}$ ) protein was expressed in E. coli B21(DE3) at $25^{\circ} \mathrm{C}$ for $48 \mathrm{~h}$ in autoinduction Terrific broth (TB) medium. The cells were harvested by 
343 (Union-Biotech co,.LTD), the supernatant was collected after centrifuged at $12,000 \times \mathrm{g}$ at $4{ }^{\circ} \mathrm{C}$

344 for $60 \mathrm{~min}$. The proteins were purified using Ni-NTA agarose resin (Qiagen), washed with 20

$345 \mathrm{mM}$ Tris- $\mathrm{HCl}, 150 \mathrm{mM} \mathrm{NaCl}, 20 \mathrm{mM}$ imidazole, $\mathrm{pH} 8.0$, and then eluted with $300 \mathrm{mM}$

346 imidazole. The Vip3 $\mathrm{Aa}_{200-e n d}$ proteins were further purified by HiTrap Q HP ion-exchange

347 chromatography and Superdex 200 gel filtration chromatography (GE Healthcare Life

348 Sciences). Fractions containing the Vip3 $\mathrm{Aa}_{200 \text {-end }}$ protein were concentrated to $\sim 7 \mathrm{mg} / \mathrm{ml}$ for

349 crystallization. The expression and purification steps of other Vip3Aa truncations were the same

350 as those of Vip3 $\mathrm{Aa}_{200-\text { end. }}$

351 SeMet-substituted Vip3Aa 200 -end was expressed in E. coli B834(DE3) strain. Briefly, the cells were cultured in the LB medium at $37{ }^{\circ} \mathrm{C}$ with shaking until the OD600 of the bacterial culture reached 1.0. The cells were harvested by centrifugation at $4000 \times \mathrm{g}$ at $4{ }^{\circ} \mathrm{C}$ for $15 \mathrm{~min}$ and the pellet was washed one time with PBS. The pellet was resuspended in 1 L Medium A (M9 medium plus) and incubated for 3 hours at $37{ }^{\circ} \mathrm{C}$. Added $50 \mathrm{mg}$ seleno-methionine in the medium and incubated for a further 30 minutes. The protein was incubated to express for a further 10 hours by adding $200 \mathrm{mM}$ IPTG (isopropyl- $\beta$-D-thiogalactopyranoside). The SeMetVip3 $\mathrm{Aa}_{200-\text { end }}$ was purified by the same procedure as for the native Vip3 $\mathrm{Aa}_{200}$-end protein.

\section{Crystallization and data collection}

The purification of $\mathrm{His}_{6}$-tagged MBP-Vip3 $\mathrm{Aa}_{200-\text { end }}$ used for crystallization is described above.

361 MBP-Vip3Aa 200 -end $(5 \mathrm{mg} / \mathrm{mL})$ was used to perform initial spare matrix crystal screening with a crystallization robot. After crystal optimization trials, MBP-Vip3Aa 200 -end $(7 \mathrm{mg} / \mathrm{mL})$ crystals grew in 3 days at $18^{\circ} \mathrm{C}$ using the hanging-drop vapor-diffusion method in a mix of $1 \mu \mathrm{l}$ of 

crystals grew in the similar condition.

A native data set with the space group of $\mathrm{P} 2{ }_{1} 2_{1} 2_{1}$ was collected at $3.62 \AA$ (native I). A weak selenomethionine (SeMet) derivative data set was collected at $3.9 \AA$ with the same symmetry as the native I crystal for the amino-acid assignment using the difference Fourier map of the SeMet derivative. After further crystallization optimization, another native crystal (native II) was obtained with the space group of $\mathrm{P} 2_{1}$ that could diffract to around $3.2 \AA$. Diffraction data were collected on BL17U1 and BL18U beamlines at Shanghai Synchrotron Radiation Facility (Shanghai, China) and processed by HKL2000 52 .

\section{Structural determination and refinement}

Molecular replacement was carried out to identity the MBP positions in the native crystals by PHASER $^{53}$. The initial phases were further improved with the multi-crystal averaging ${ }^{54}$. Model building was performed manually in $\mathrm{COOT}^{55}$, and the sequence assignment was helped with the SeMet anomalous difference map. Figures were prepared using PyMol (v.2.3.2, https://pymol.org/). Structure refinement was done by PHENIX ${ }^{56}$. The data collection and refinement statistics are summarized in Supplementary Table 1.

\section{Glycan microarray analysis}

Purified full-length Vip3Aa protein were labelled with EZ-Link NHS-LC-LC-Biotin reagent in $20 \mathrm{mM}$ HEPES pH 8.0 and $100 \mathrm{mM} \mathrm{NaCl}$ with a 4:1 molar excess of biotin. Unreacted biotin was removed by dialysis, and the resulting protein was concentrated by Amicon Ultra centrifugal filter (Millipore). The sample was diluted to $2.5 \mu \mathrm{g} / \mathrm{ml}$ and analyzed on a glycan 100 binding array provided by Creative Biochip. The reaction buffer was used as the negative 
control and the streptavidin was used as the positive control. The signals were measured in microarray scanner (LuxScan-10K/A; CapitalBio). The mean signal representing one glycan probe is the average of signals from triplicate spots on a single glycan array and compared by one-way ANOVA with Dunnett's multiple comparisons test. All assays were repeated at least three times.

\section{Immunofluorescence}

393 Sf9 cells with a density of $5 \times 10^{4}$ cells per $\mathrm{ml}$ were seeded into 6 -well culture plates separately.

394 After overnight culture, the cells were respectively treated with RFP tagged Vip3Aa or its truncations $(0.15 \mu \mathrm{M})$ for $6 \mathrm{~h}$. After treatment, the cells were washed three times with PBS to remove unbound proteins, and fixed with $4 \%$ paraformaldehyde at $37{ }^{\circ} \mathrm{C}$ for $15 \mathrm{~min}$. The cell nuclei were labeled with DAPI $(0.2 \mu \mathrm{g} / \mathrm{ml})$ for $30 \mathrm{~min}$. Cell images were captured using a Nikon.

TI-E inverted fluorescence Microscope.

\section{Statistical analysis}

401 All functional assays were performed at least three times independently. Data were shown as means \pm SD. All statistical data were calculated using GraphPad Prism version 8.0. One-way ANOVA followed by Dunnett's test were used to identify statistically significant differences between treatments. Significance of mean comparison is annotated as follow: ns, not significant; 


\section{References}

410 1. Tabashnik, B.E. \& Carrière Y. Surge in insect resistance to transgenic crops and prospects for sustainability. Nat. Biotechnol. 35, 926-935 (2017).

2. Jouzani, G.S., Valijanian, E. \& Sharafi, R. Bacillus thuringiensis: a successful insecticide with new environmental features and tidings. Appl. Environ. Microbiol. 101, 2691-2711 (2017).

4. Adang, M.J., Crickmore, N. \& Jurat-Fuentes, J.L. Diversity of Bacillus thuringiensis crystal toxins and mechanism of action. Adv. In. Insect. Phys. 47, 39-87. (2014).

5. Jurat-Fuentes, J.L. \& Crickmore, N. Specificity determinants for Cry insecticidal proteins:

6. de Almeida Melo, A.L., Soccol, V.T. \& Soccol, C.R. Bacillus thuringiensis: mechanism of action, resistance, and new applications: a review. Crit. Rev. Biotechnol. 36, 317-326 (2016).

8. Tabashnik, B.E. \& Carrière, Y. Global patterns of resistance to Bt crops highlighting Pink

9. Chakroun, M., Banyuls, N., Bel, Y., Escriche, B. \& Ferré J. Bacterial Vegetative Insecticidal Proteins (Vip) from Entomopathogenic Bacteria. Microbiol. Mol. Biol. Rev. 80, 329-350 (2016).

11. Lee, M.K., Walters, F.S., Hart, H., Palekar, N. \& Chen, J.S. Mode of action of the Bacillus

12. Sena, J.A.D., Sara Hernández-Rodríguez, C. \& Ferré J. Interaction of Bacillus thuringiensis

13. Jiang, K. et al. Fibroblast Growth Factor Receptor, a Novel Receptor for Vegetative Insecticidal

14. Jiang, K. et al. Scavenger receptor-C acts as a receptor for Bacillus thuringiensis vegetative insecticidal protein Vip3Aa and mediates the internalization of Vip3Aa via endocytosis. PloS. Pathog. 14 (2018).

17. Chen, W.-b. et al. Transgenic cotton coexpressing Vip3A and Cry1Ac has a broad insecticidal synergizes their toxicity against Asiatic rice borer (Chilo suppressalis). J. Biol. Chem. 293, 
11447-11458 (2018).

19. Xiao, Y. \& Wu, K. Recent progress on the interaction between insects and Bacillus thuringiensis crops. Philoso. T. R. Soc. B. 374 (2019).

20. Bel, Y., Banyuls, N., Chakroun, M., Escriche, B. \& Ferré J. Insights into the structure of the Vip3Aa insecticidal protein by protease digestion analysis. Toxins 9 (2017).

21. Kunthic, T., Surya, W., Promdonkoy, B., Torres, J. \& Boonserm, P. Conditions for homogeneous preparation of stable monomeric and oligomeric forms of activated Vip3A toxin from Bacillus thuringiensis. Eur. Biophys. J .Biophy. 46, 257-264 (2017).

22. Gayen, S., Hossain, M.A. \& Sen, S.K. Identification of the bioactive core component of the insecticidal Vip3A toxin peptide of Bacillus thuringiensis. J. Plant. Biochem. Biot. 21, S128S135 (2012).

23. Zack, M.D. et al. Functional characterization of Vip3Ab1 and Vip3Bc1: Two novel insecticidal proteins with differential activity against lepidopteran pests. Sci. Rep. 7 (2017).

24. Abdelkefi-Mesrati, L. et al. Investigation of the steps involved in the difference of susceptibility of Ephestia kuehniella and Spodoptera littoralis to the Bacillus thuringiensis Vip3Aa16 toxin. J. Invertebr. Pathol. 107, 198-201 (2011).

25. Chakroun, M. \& Ferré J. In vivo and In vitro binding of Vip3Aa to Spodoptera frugiperda midgut and characterization of binding sites by I-125 radiolabeling. Appl. Environ. Microbiol. 80, 6258-6265 (2014).

26. Zhang, L. et al. Proteolytic activation of Bacillus thuringiensis Vip3Aa protein by Spodoptera exigua midgut protease. Int. J. Biol. Macromol. 107, 1220-1226 (2018).

27. Palma, L. et al. The Vip3Ag4 insecticidal protoxin from Bacillus thuringiensis adopts a tetrameric configuration that is maintained on proteolysis. Toxins 9 (2017).

28. Quan, Y. \& Ferré J. Structural domains of the Bacillus thuringiensis Vip3Af protein unraveled by tryptic digestion of alanine mutants. Toxins 11 (2019).

29. Estruch, J.J. et al. Vip3A, a novel Bacillus thuringiensis vegetative insecticidal protein with a wide spectrum of activities against lepidopteran insects. Proc. Natl. Acad. Sci. U S A. 93, 53895394 (1996).

30. Jiang, K. et al. Vip3Aa induces apoptosis in cultured Spodoptera frugiperda (Sf9) cells. Toxicon 120, 49-56 (2016).

31. Hernández-Martínez, P., Gomis-Cebolla, J., Ferré J. \& Escriche, B. Changes in gene expression and apoptotic response in Spodoptera exigua larvae exposed to sublethal concentrations of Vip3 insecticidal proteins. Sci. Rep. 7 (2017).

32. Banyuls, N., Hernández-Rodríguez, C.S., Van Rie, J. \& Ferré J. Critical amino acids for the insecticidal activity of Vip3Af from Bacillus thuringiensis: Inference on structural aspects. Sci. Rep. 8 (2018).

33. Sellami, S. et al. A novel Vip3Aa16-Cry1Ac chimera toxin: Enhancement of toxicity against Ephestia kuehniella, structural study and molecular docking. Int. J. Biol. Macromol. 117, 752761 (2018).

34. Krissinel, E. \& Henrick, K. Inference of macromolecular assemblies from crystalline state. $J$. Mol. Biol. 372, 774-797 (2007).

35. Holm, L. Benchmarking fold detection by DaliLite v.5. Bioinformatics (Oxford, England) (2019).

36. Dal Peraro, M. \& van der Goot, F.G. Pore-forming toxins: ancient, but never really out of 
fashion. Nat. Rev. Microbiol. 14, 77-92 (2016).

37. Singh, G., Sachdev, B., Sharma, N., Seth, R. \& Bhatnagar, R.K. Interaction of Bacillus thuringiensis vegetative insecticidal protein with ribosomal S2 protein triggers larvicidal activity in Spodoptera frugiperda. Appl. Environ. Microbiol. 76, 7202-7209 (2010).

38. Bae, B. et al. Molecular basis for the selectivity and specificity of ligand recognition by the family 16 carbohydrate-binding modules from Thermoanaerobacterium polysaccharolyticum ManA. J. Biol. Chem. 283, 12415-12425 (2008).

39. Hegedus, D.D., Toprak, U. \& Erlandson, M. Peritrophic matrix formation. J. Insect. Physiol 117, 103898 (2019).

40. Liu, X., Cooper, A.M.W., Zhang, J. \& Zhu, K.Y. Biosynthesis, modifications and degradation of chitin in the formation and turnover of peritrophic matrix in insects. J. Insect. Physiol 114, 109-115 (2019).

41. Yu, C.G., Mullins, M.A., Warren, G.W., Koziel, M.G. \& Estruch, J.J. The Bacillus thuringiensis vegetative insecticidal protein Vip3A lyses midgut epithelium cells of susceptible insects. Appl. Environ. Microbiol 63, 532-536 (1997).

42. Grochulski, P. et al. Bacillus thuringiensis cryla(a) insecticidal toxin-crystal-structure and channel formation. J. Mol. Biol. 254, 447-464 (1995).

43. Li, J.D., Carroll, J. \& Ellar, D.J. Crystal-structure of insecticidal $\delta$-endotoxin from Bacillus thuringiensis at $2.5 \AA$ resolution. Nature 353, 815-821 (1991).

44. Schwartz, J.L. et al. Restriction of intramolecular movements within the Cry1 Aa toxin molecule of Bacillus thuringiensis through disulfide bond engineering. FEBS. Lett. 410, 397-402 (1997).

45. Gazit, E., La Rocca, P., Sansom, M.S.P. \& Shai, Y. The structure and organization within the membrane of the helices composing the pore-forming domain of Bacillus thuringiensis deltaendotoxin are consistent with an "umbrella-like" structure of the pore. Proc. Natl. Acad. Sci. U S A. 95, 12289-12294 (1998).

46. de Maagd, R.A. et al. Domain III of the Bacillus thuringiensis delta-endotoxin Cry1Ac is involved in binding to Manduca sexta brush border membranes and to its purified aminopeptidase N. Mol. Microbiol. 31, 463-471 (1999).

47. Jenkins, J.L., Lee, M.K., Valaitis, A.P., Curtiss, A. \& Dean, D.H. Bivalent sequential binding model of a Bacillus thuringiensis toxin to gypsy moth aminopeptidase N receptor. J. Biol. Chem. 275, 14423-14431 (2000).

48. Jurat-Fuentes, J.L. \& Adang, M.J. Characterization of a Cry1 Ac-receptor alkaline phosphatase in susceptible and resistant Heliothis virescens larvae. Eur. J. Biochem. 271, 3127-3135 (2004).

49. Sengupta, A., Sarkar, A., Priya, P., Dastidar, S.G. \& Das, S. New insight to structure-function relationship of GalNAc mediated primary interaction between insecticidal Cry1Ac toxin and HaALP receptor of Helicoverpa armigera. PloS One. 8 (2013).

50. Zheng, M., Evdokimov, A.G., Moshiri, F., Lowder, C. \& Haas, J. Crystal structure of a Vip3B family insecticidal protein reveals a new fold and a unique tetrameric assembly. Protein. Sci. (2019).

51. Gibson, D.G. et al. Enzymatic assembly of DNA molecules up to several hundred kilobases. Nat. Methods 6, 343-345 (2009).

52. Otwinowski, Z. \& Minor, W. Processing of X-ray diffraction data collected in oscillation mode. Macromolecular Crystallography, Pt A 276, 307-326 (1997).

53. McCoy, A.J. et al. Phaser crystallographic software. J. Appl. Crystallogr. 40, 658-674 (2007). 
540 54. Aver, N. \& Skeletonisation, S. K. Cowtan, Joint CCP4 and ESF-EACBM Newsletter on Protein Crystallography, 31, p34-38. Csb.yale.edu (1994).

55. Emsley, P. \& Cowtan, K. Coot: model-building tools for molecular graphics. Acta. Crystallogr. D. 60, 2126-2132 (2004).

56. Adams, P.D. et al. PHENIX: a comprehensive Python-based system for macromolecular structure solution. Acta. Crystallogr. D. 66, 213-221 (2010).

$54657 . \quad$ Larkin, M.A. et al. Clustal W and clustal X version 2.0. Bioinformatics 23, 2947-2948 (2007).

$54758 . \quad$ Robert, X. \& Gouet, P. Deciphering key features in protein structures with the new ENDscript server. Nucleic. Acids. Res. 42, W320-W324 (2014). 


\section{Data availability}

568 Coordinate for the atomic structure has been deposited in the RCSB Protein Data Bank under RCSB ID XXX. The data that support the findings of this study are available

570 from the corresponding author upon reasonable request.

\section{Acknowledgements}

572 We thank Jiawei Wang for providing the suggestion for structure determination, Casey Flower

573 and Jorge Galan for constructive proofreading of this manuscript, the staffs from BL17U1/BL18U/BL19U1 beamlines of National Facility for Protein Science Shanghai (NFPS) at Shanghai Synchrotron Radiation Facility (SSRF) for assistance during data collection, and Xiaoju Li from Shandong University Core facilities for life and environmental sciences for her help with the XRD. This work was supported by the National Natural Science Foundation of 
Fig. 1 Overall structure of Vip3Aa11 200-end. a Domain organization of Vip3A. b Two views of the overall structure of Vip3Aa11 200 -end monomer coloured as in a. $\mathbf{c}$ Two views of the surface model of Vip3Aa11 200-end monomer coloured as in $\mathbf{a}$.

Fig. 2 Domain II of Vip3Aa11 shows a conserved hydrophobic surface. a Two views of structure of Vip3Aa11 domain II shown as a ribbon cartoon. b Two views of the surface model of helix $\alpha 4$ from domain II show its surface charge distribution. $\mathbf{c}$ The highly conserved amino acid residues from Vip3 family sequence alignment (Supplementary Figure 4) are highlighted in the Vip3Aa toxic core structure with red color. d Two views of the surface model of Vip3Aa11 domain II show its surface charge distribution. The conserved hydrophobic surface is highlighted by black square. $\mathbf{b}, \mathbf{d}$ The surface is coloured as the basis of electrostatic potential with positive charged surface in blue and negatively charged area in red. are shown in three different colors. b Two views of the surface model of domain III of Vip3 Aa11. amino acid residues from three antiparallel $\beta$ sheets are shown as sticks. $\mathbf{c}$ The schematics of images of Sf9 cells treated with Vip3Aa-RFP or its truncations, which were labeled with C- 
611 of Sf9 cells that can be bound by RFP-tagged Vip3Aa and its truncations of Fig3 $\mathbf{d}$ in a blind

612 fashion $(n=100$ cells per sample). Data are expressed as the mean \pm SD from three independent

613 experiments.

Fig. 4 Domains IV and V of Vip3Aa11 have glycan binding motifs. a Domain architectures

of domains III, IV and V of Vip3Aa11. The polar interactions between domain IV, V and domain

III are shown as sticks. b Overall structure of Vip3Aa11 domain IV and V shown as a ribbon the sequence alignment ${ }^{57}$. ESPript-3.0 was used to generate the figure ${ }^{58}$. d Two views of structure superimposition between domain IV and domain V of Vip3Aa11 shown as a ribbon

RCSB ID 2ZEY) shown as a ribbon cartoon. Domains IV and V are coloured as Figure4b, and

CBM16 is shown in magenta color. The glycan in CBM16 is shown as stick in light brown color.

Fig. 5 Domains IV and V of Vip3Aa11 can recognize glycans. a Surface charge distribution 

blue, blue, light brown, magenta and green, respectively. b Structural overlay of domain II between Vip3A(green) and Vip3B(blue). The inset shows a detailed view of the conformational changes. Appreciable conformational differences are observed in helices $\alpha 1$ and $\alpha 2$. The conserved hydrophobic amino acid residues are marked in red on helices $\alpha 1$ and $\alpha 4$. The Val219 is highlighted as stick to show the rotation along its y axis of helix $\alpha 1$ during the conformational change. c A proposed membrane insertion model of Vip3A toxin. A conserved hydrophobic helical hairpin from typical colicin fold is shown in blue (left-hand side). The conserved hydrophobic helices $\alpha 1$ and $\alpha 4$ of Vip3A form a hairpin-like motif and are shown as cylindrical cartoon. Similar to the colicin family pore-forming toxin, Vip3A may insert into the host cell membrane through the hairpin-like structure from domain II. 
a
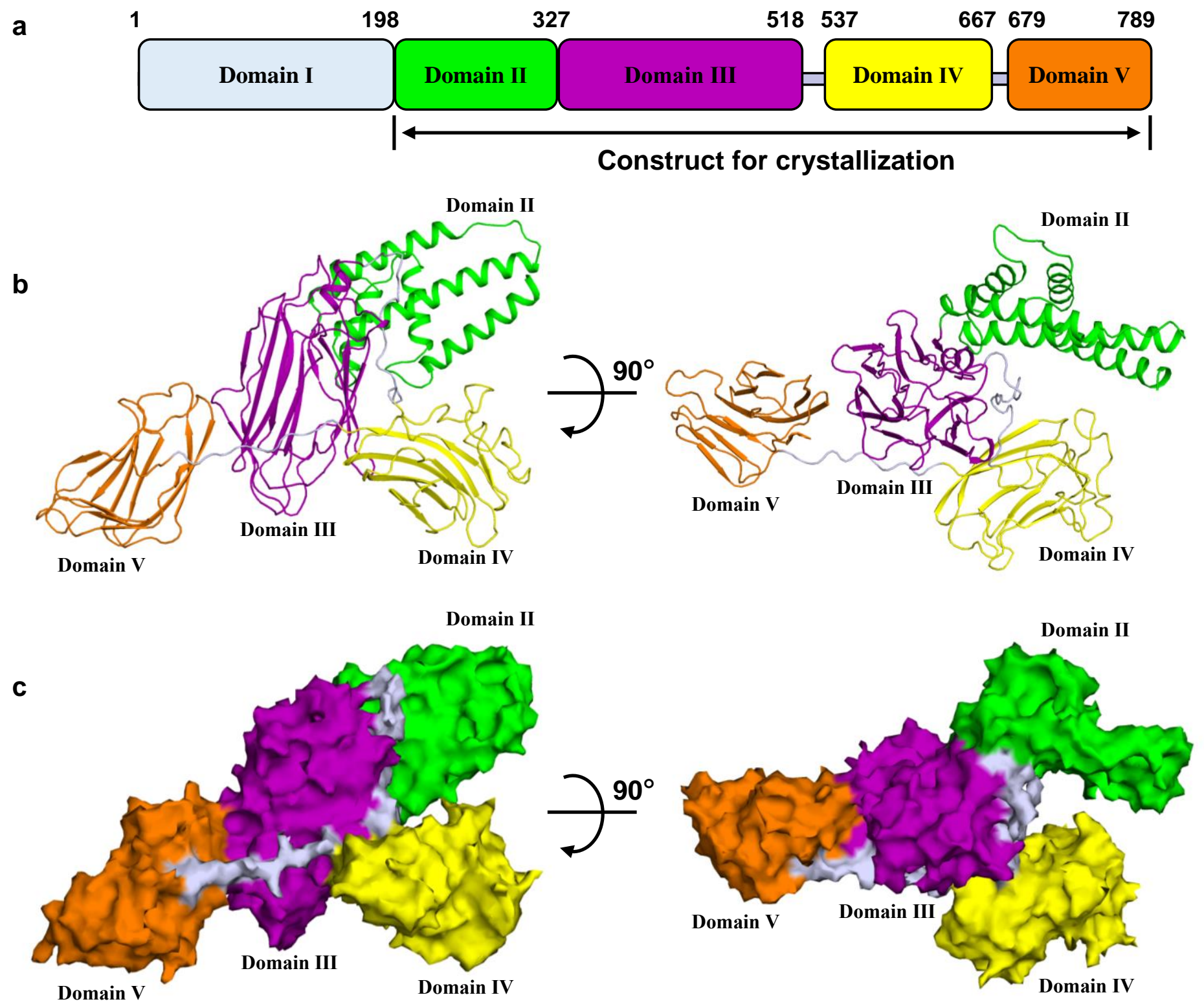
a

c
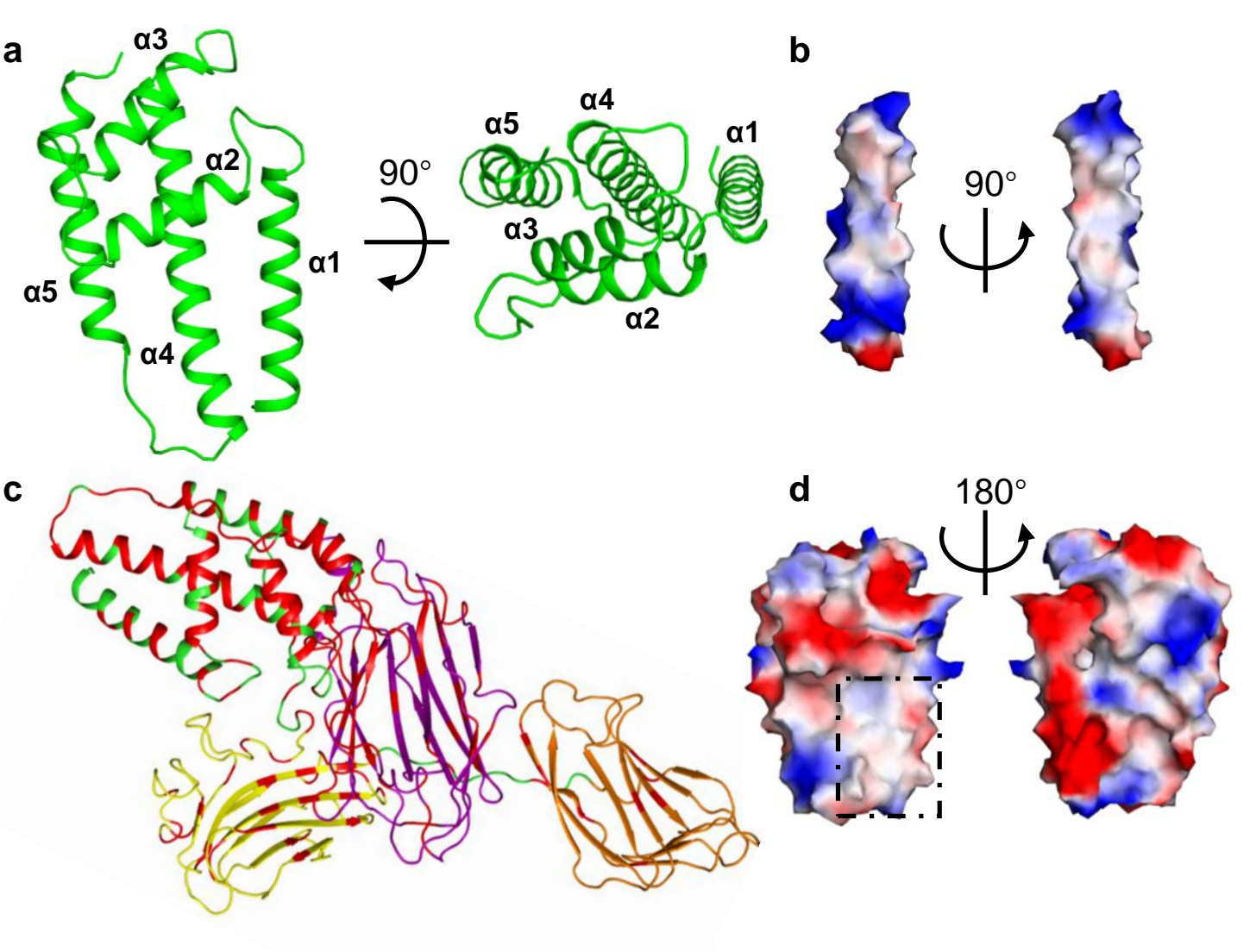

b
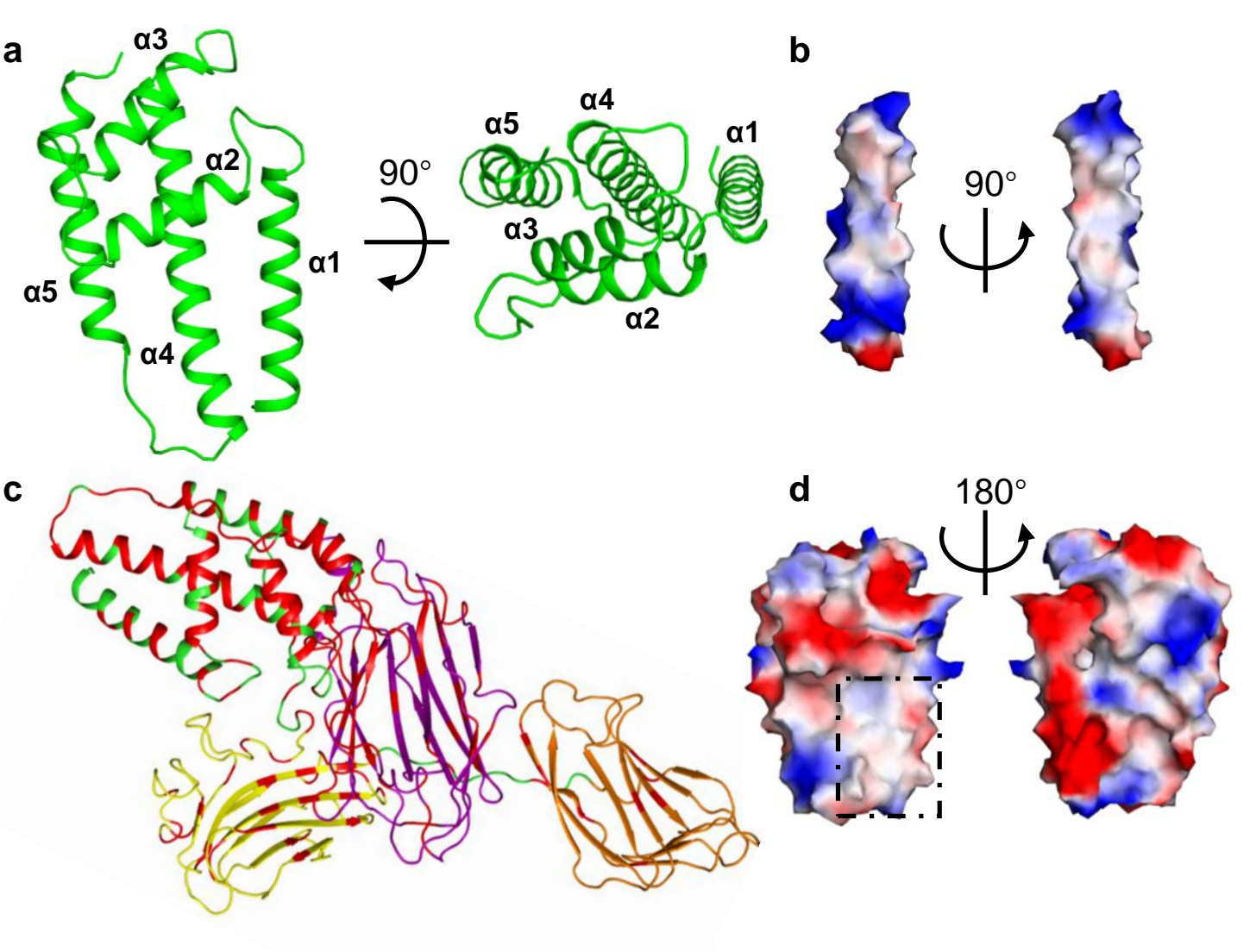

$$
\text { (1) }
$$

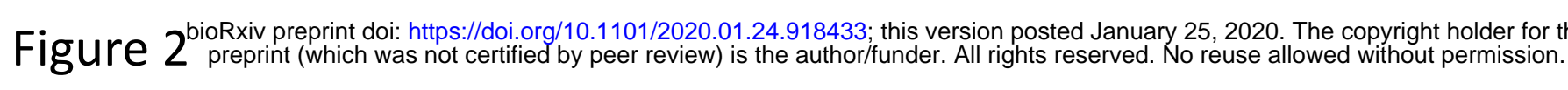

.

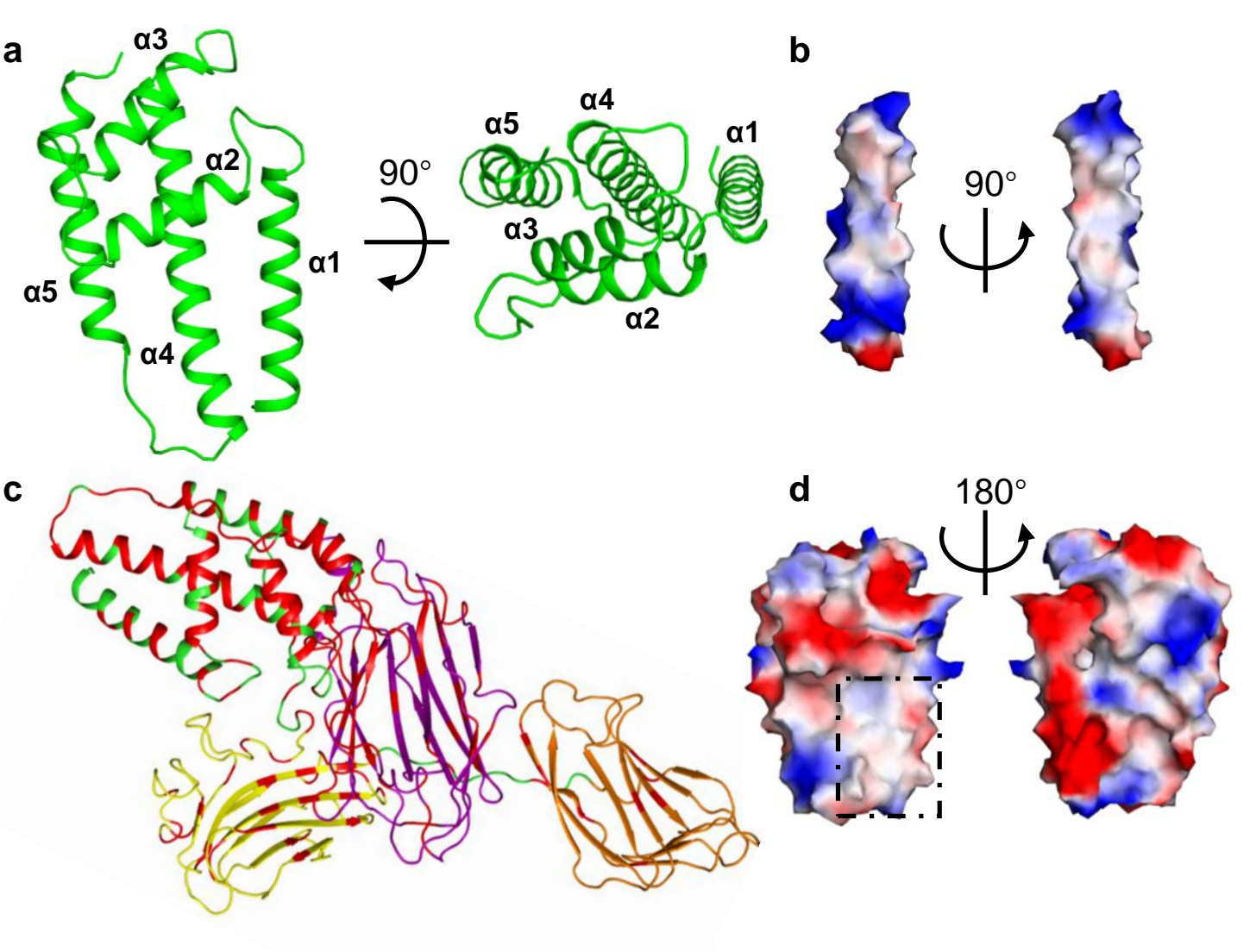

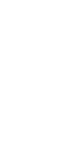

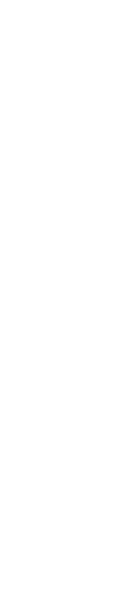


Figure 3 bioRxiv preprint doi: https://doi.org/10.1101/2020.01.24.918433; this version posted January 25, 2020. The copyright holder for this
preprint (which was not certified by peer review) is the author/funder. All rights reserved. No reuse allowed without permission.

a

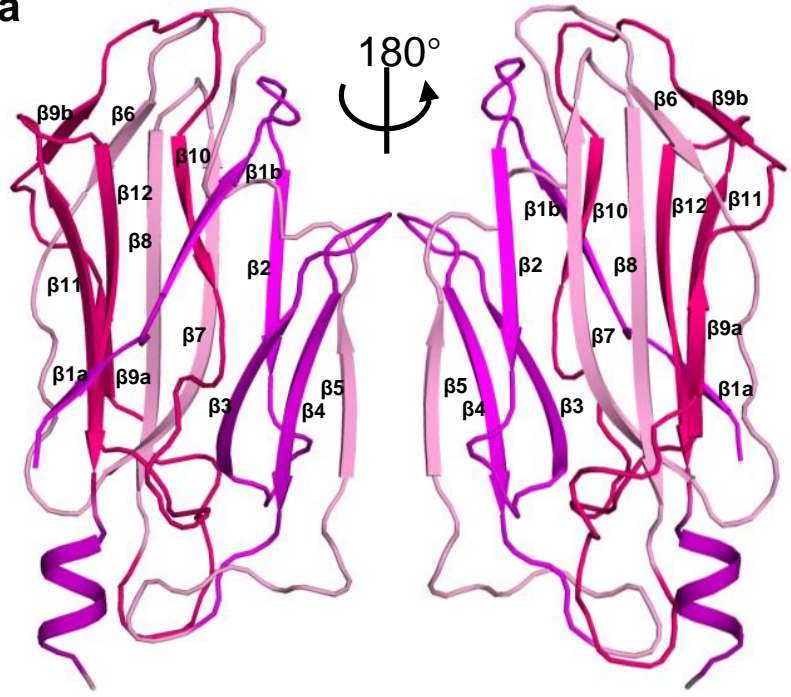

b

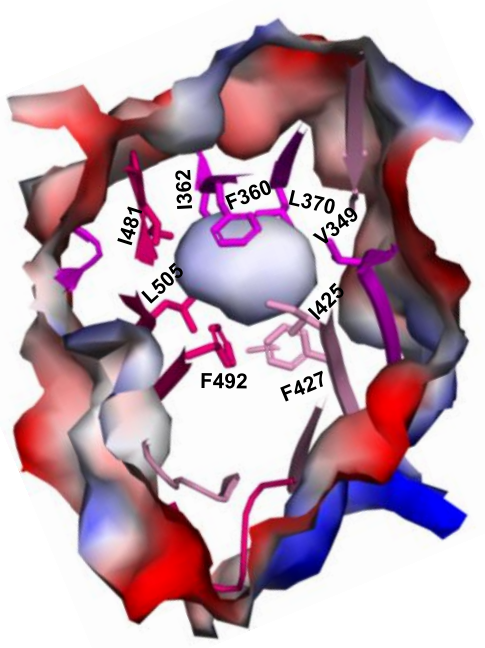

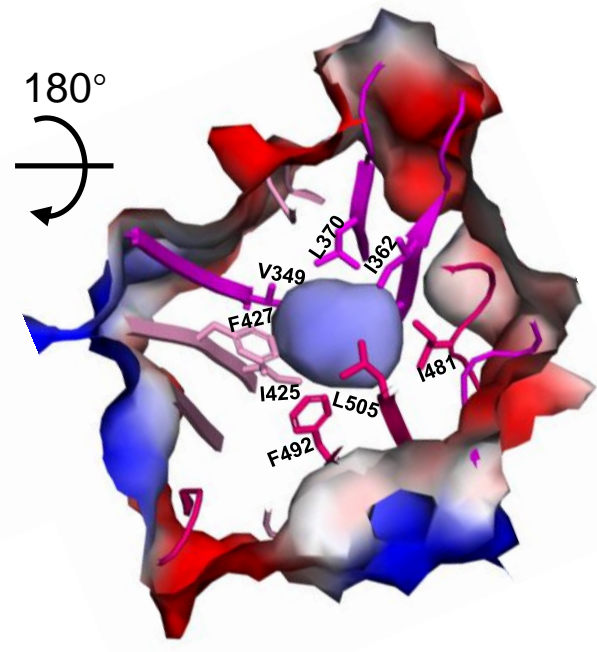

C

Vip3-Full

\begin{tabular}{|c|c|c|c|c|}
\hline Dm I & $\mathrm{Dm}$ II & Dm III & Dm IV & $\mathrm{Dm} V$ \\
\hline
\end{tabular}

Dm I-III

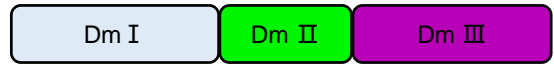

Dm IV-V

Dm IV Dm

Dm II-III

Dm II

Dm I-II

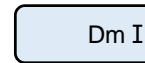

Dm II

Dm III

d

Dm-RFP

DAPI

Merge

高
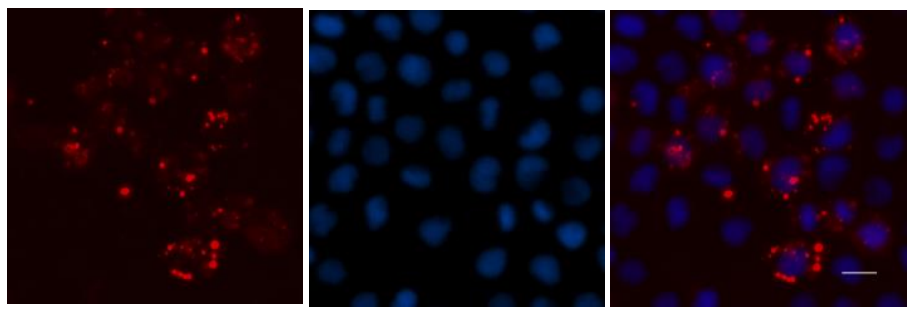

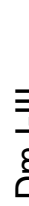
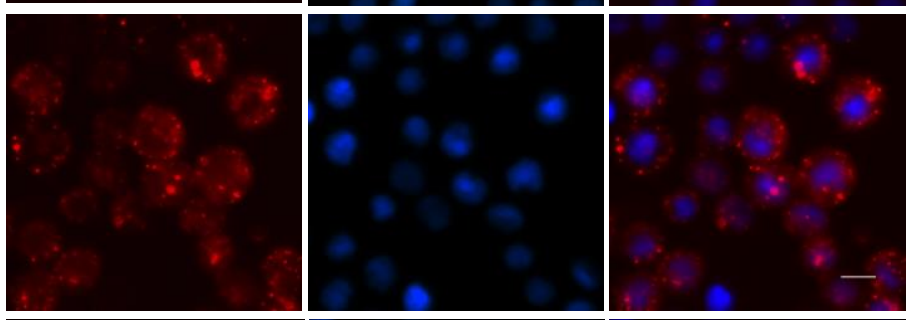

$>$
$\geq$
ำ

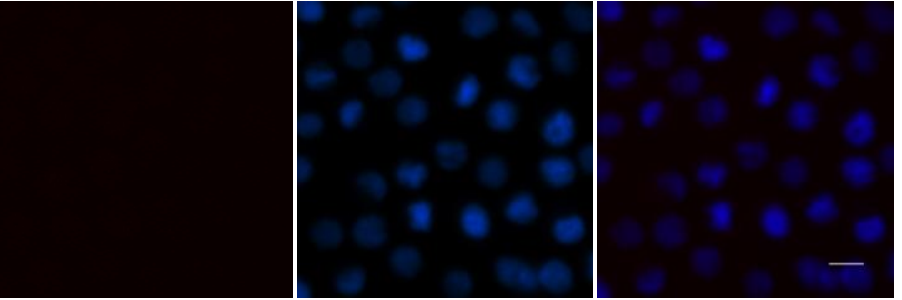

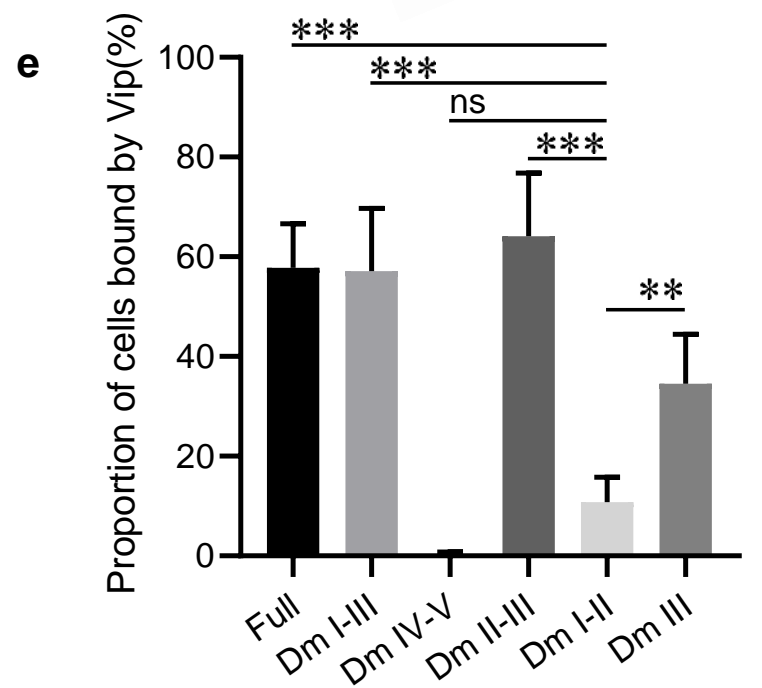

Dm-RFP

DAPI

Merge

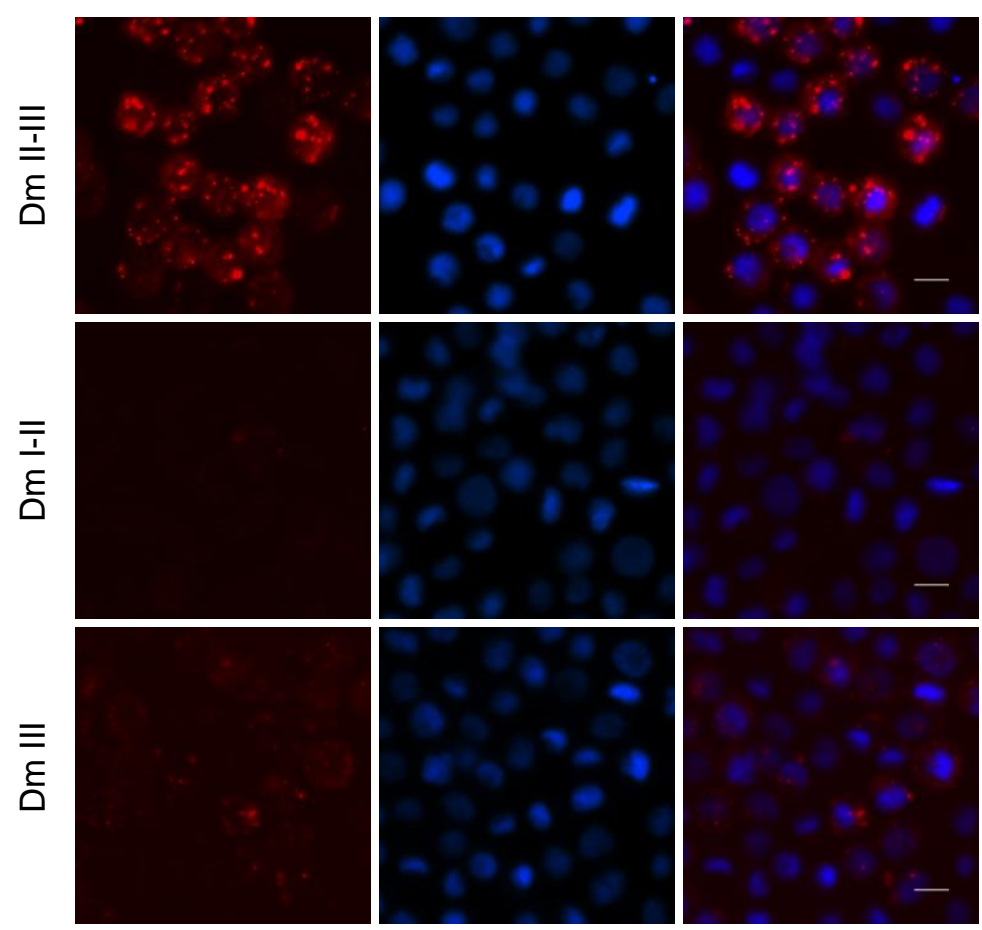




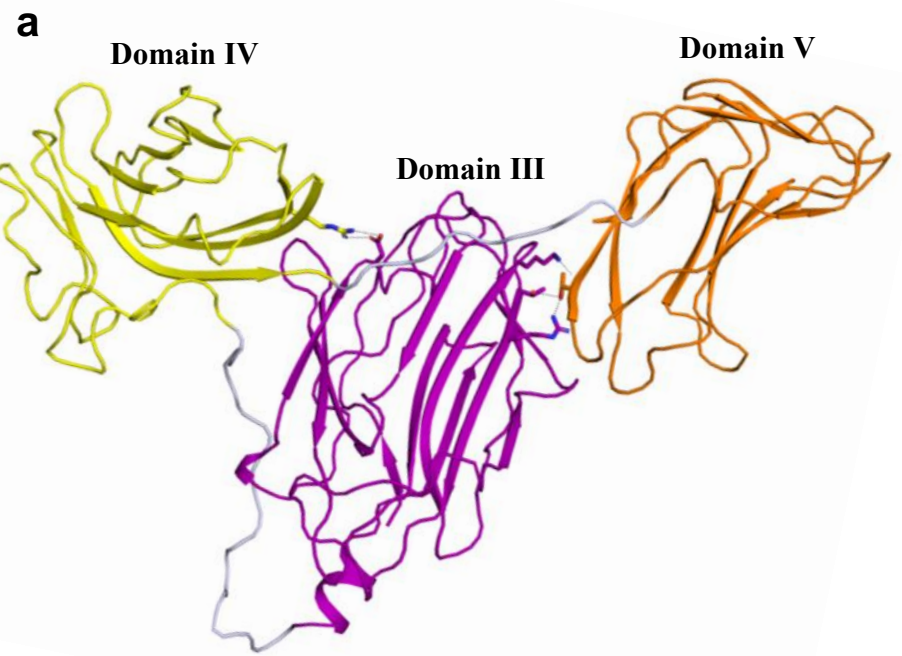

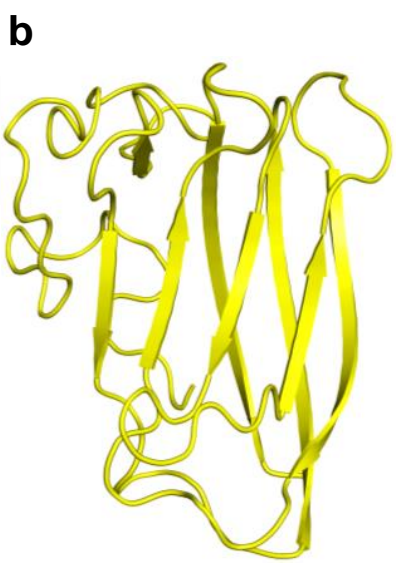

Domain IV

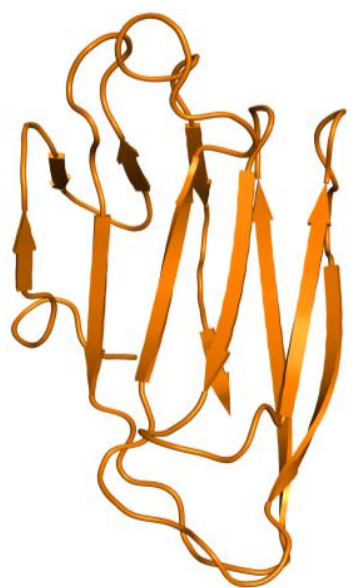

Domain V

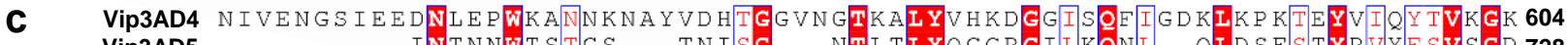

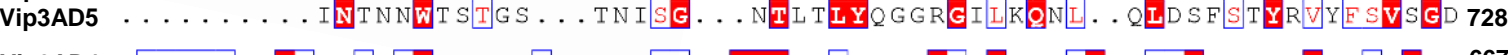

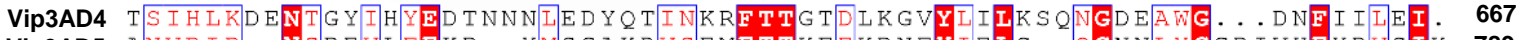

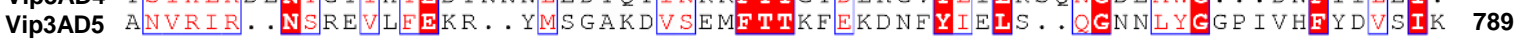

d

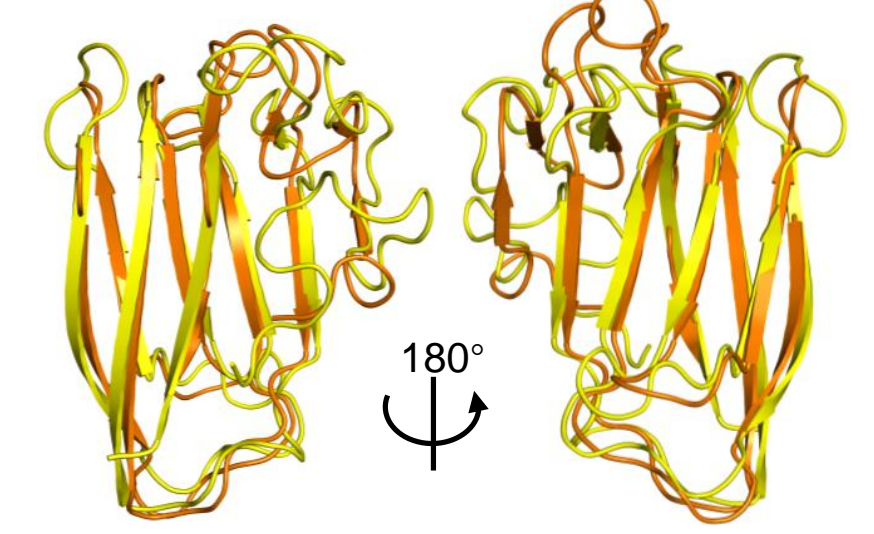

e

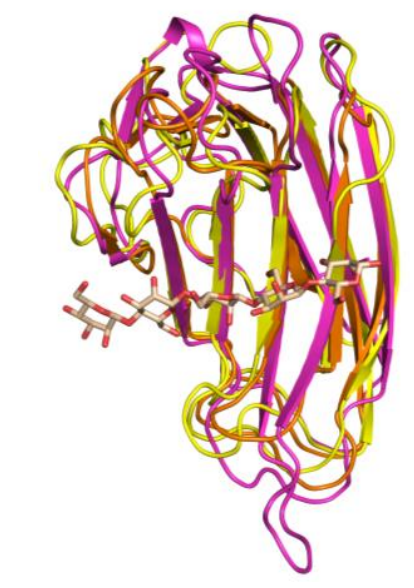


Figure 5 preprint (which was not certified by peer review) is the author/funder. All rights reserved. No reuse allowed without permission.
prignt

Figure 5 breprint (which was not certified by peer review) is the author/funder. All rights reserved. No reuse allowed without permission.

a

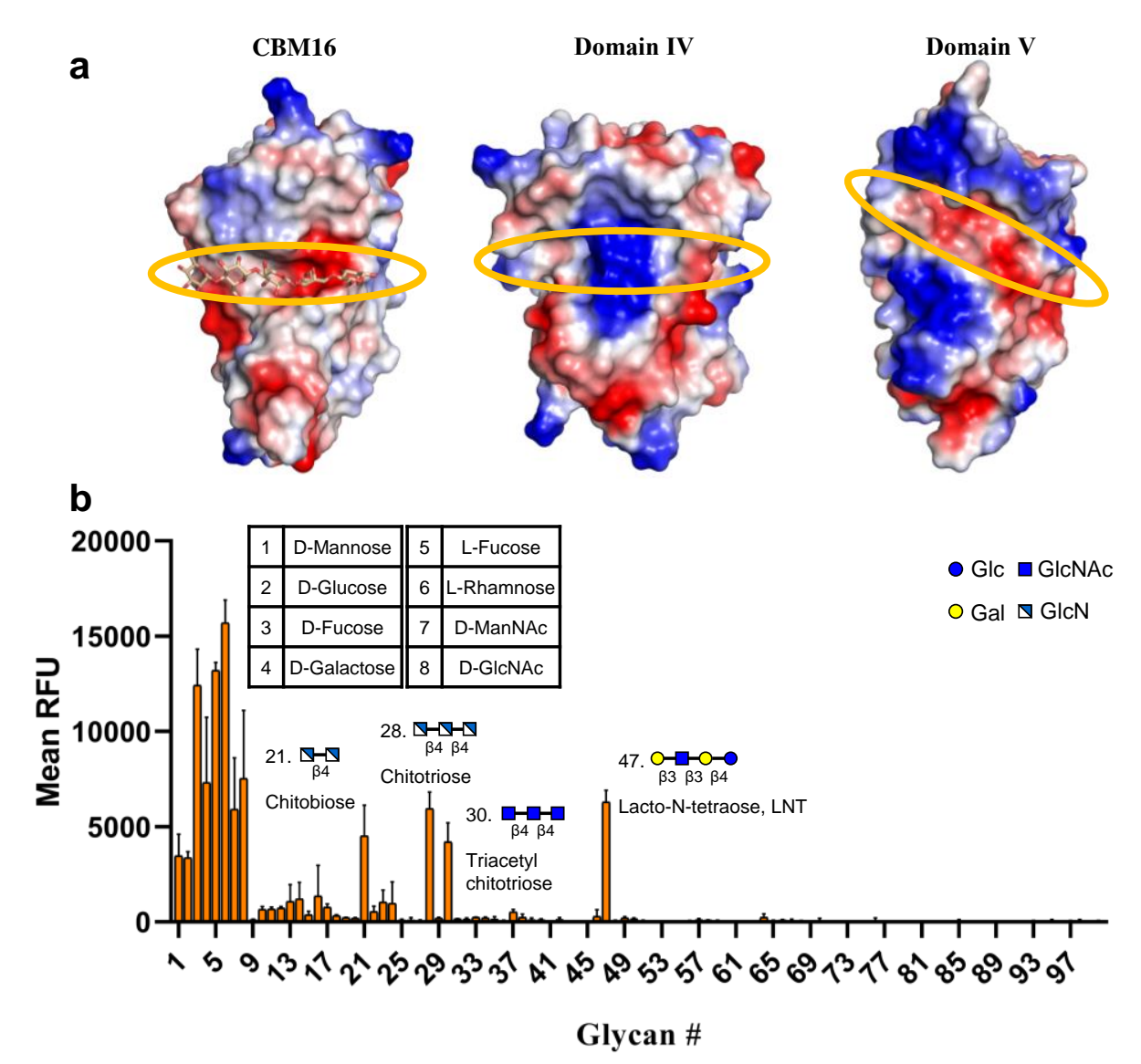

b

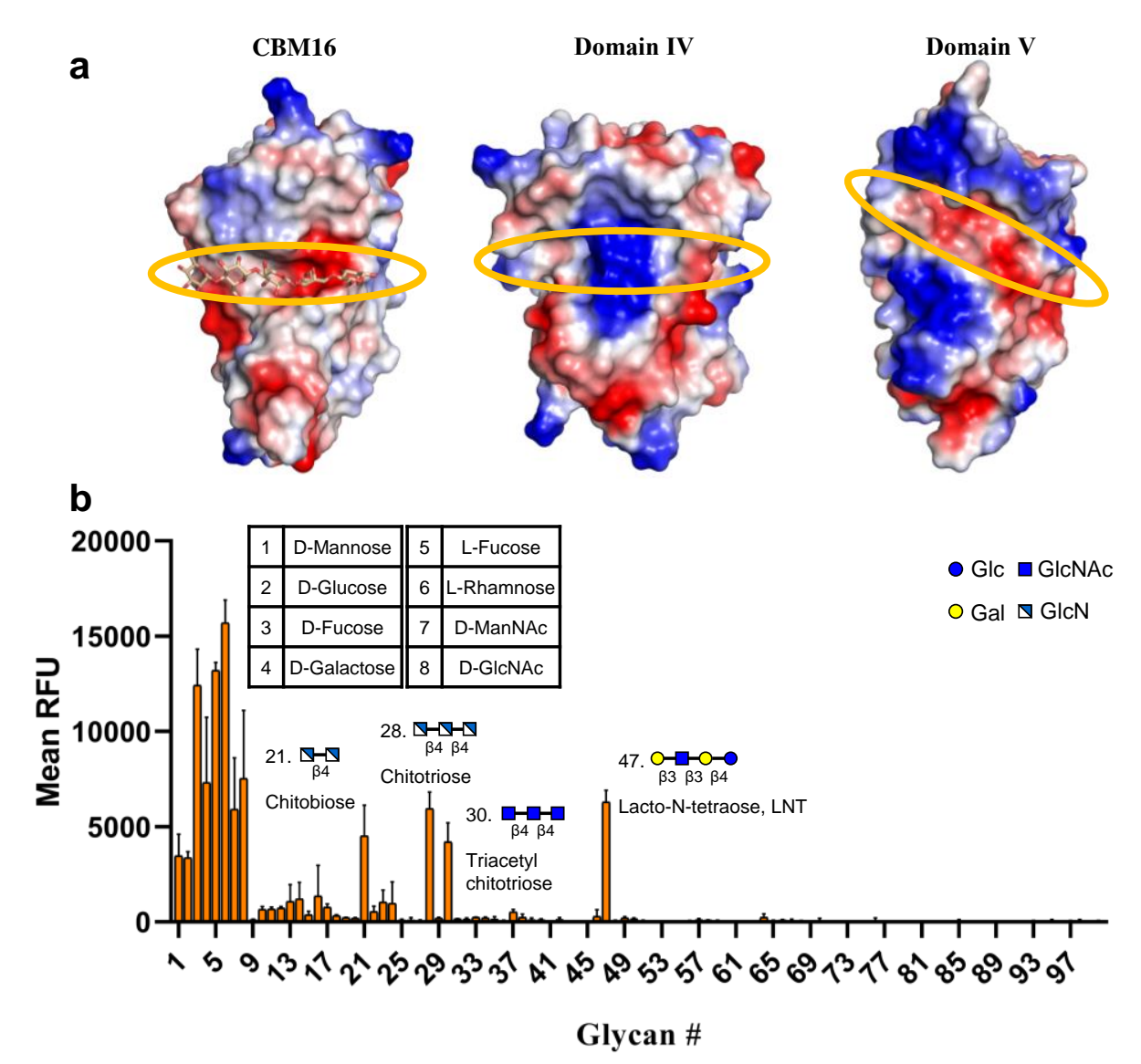

Domain IV

Glycan \#

\section{Glycan \#}
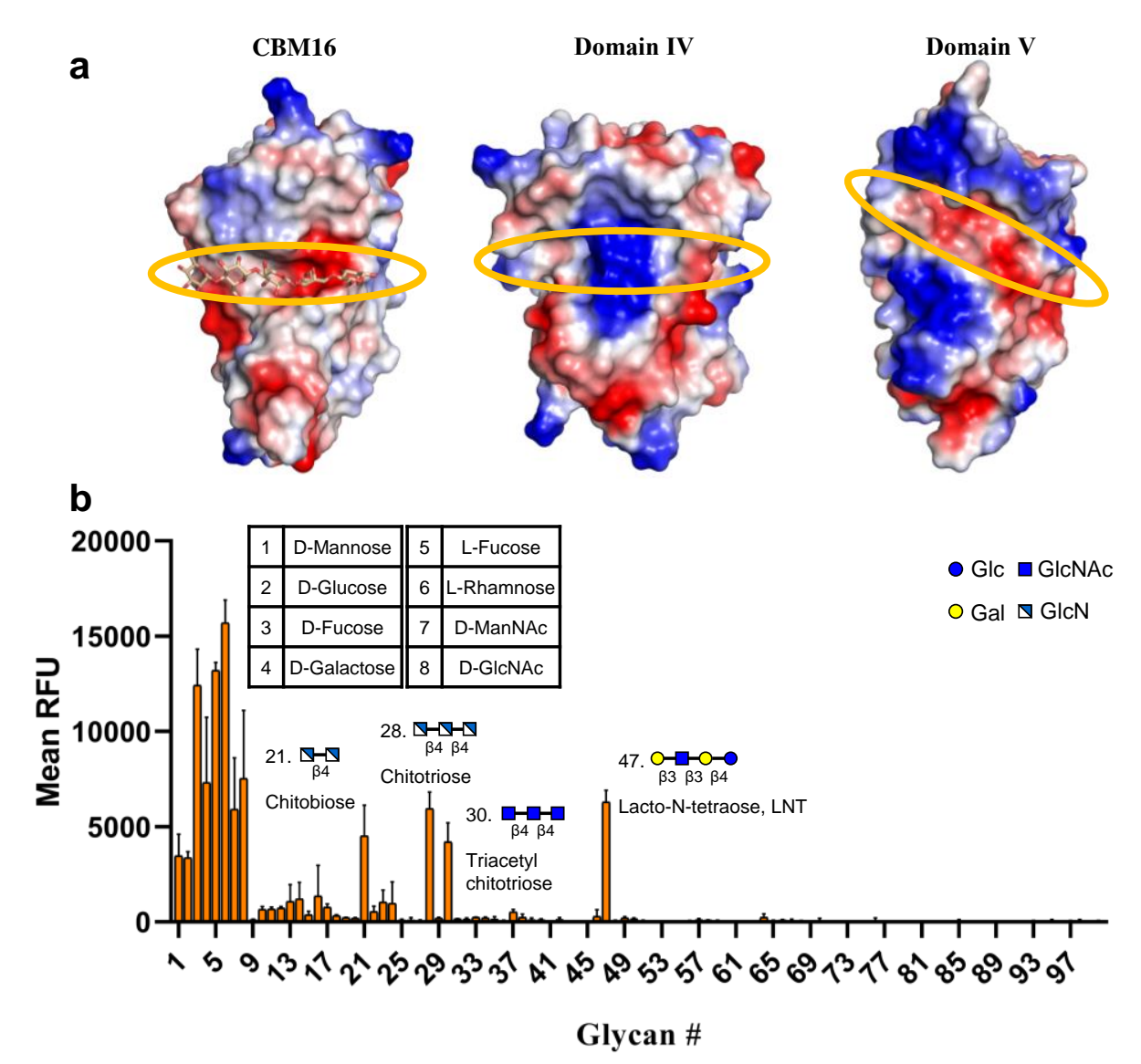

- Glc $\square$ GlcNAc

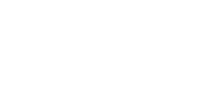

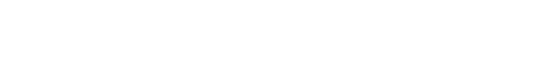

(n)

.

(2) 
Figure $6^{\text {bioRxiv preprint doi: https://doi.org/10.1101/2020.01.24.918433; this version posted January 25, 2020. The copyright holder for this }}$ Figure 6 preprint (which was not certified by peer review) is the author/funder. All rights reserved. No reuse allowed without permission.

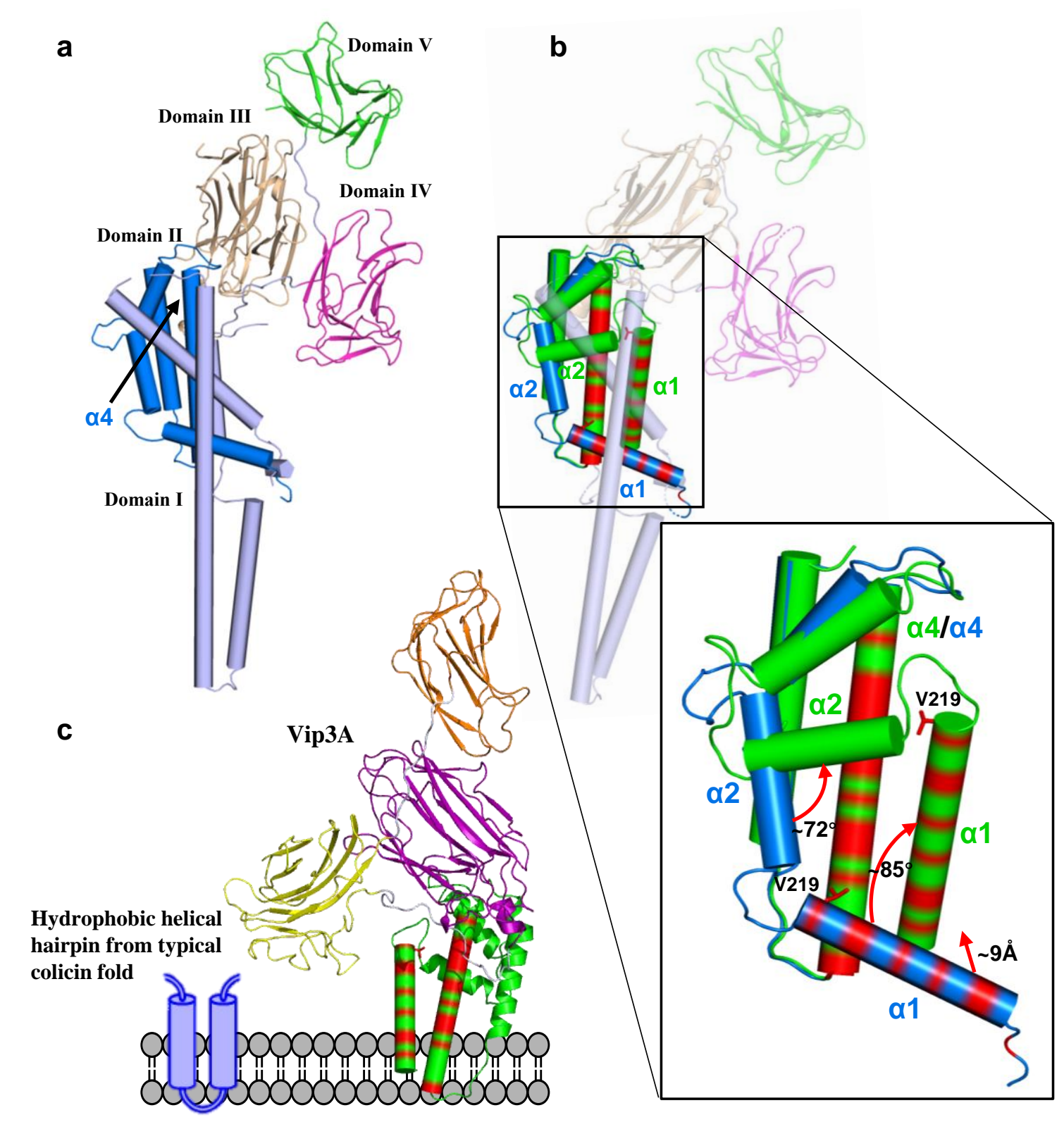

